\title{
Articula 8
}

\section{SISTEMAS CONSTRUCTIVOS INDUSTRIALIZADOS PARA RE- SOLUCION DE EQUIPAMIENTOSREN SITUACIONES DE CA- TÁSTROFE}

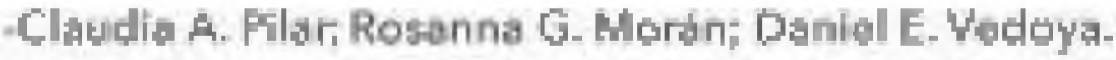

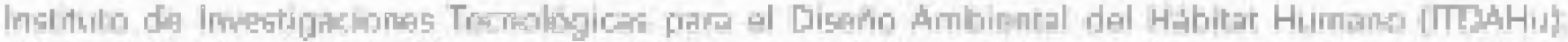

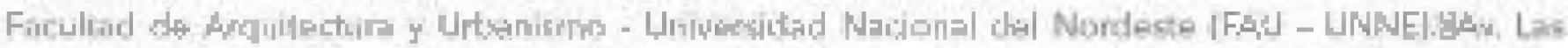

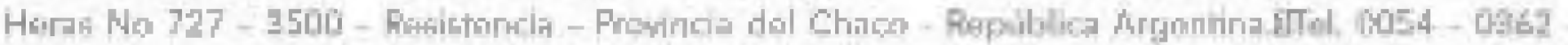

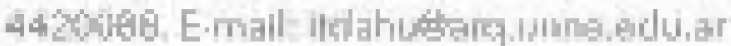

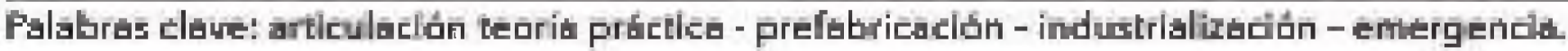

\section{Recuman:}

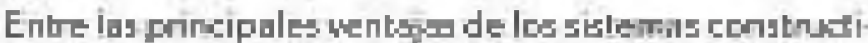

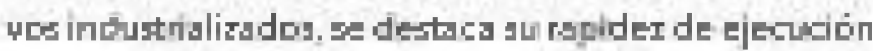

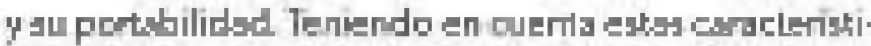

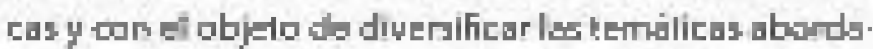

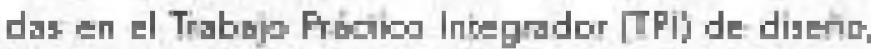
que estructura y aricula el dictado de la signalwa Contruccicnes II de la Faculted de Arquitectira $\mathrm{y}$ Urts. níme de la binisersided Nacional del Mardele, se

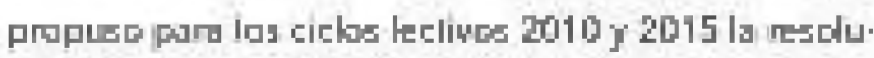

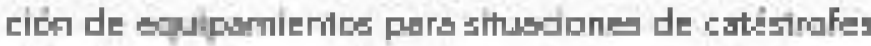
näuralas anmo sar inund-jones, tarremador, huracs.

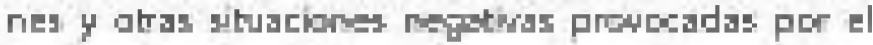
hignibue, dada que los mismas requieren de repouetag

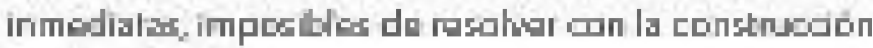
tradicions

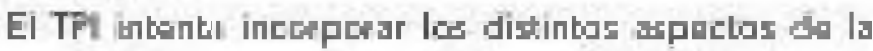

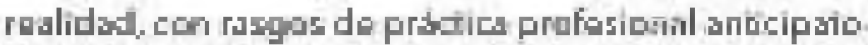

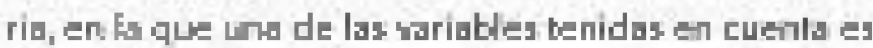

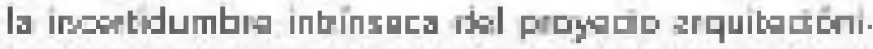

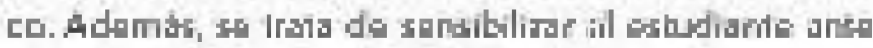

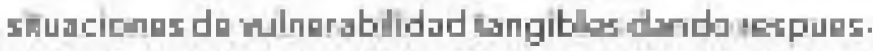

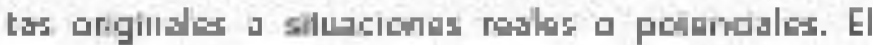

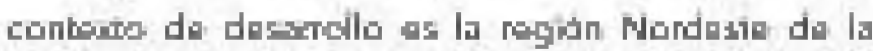

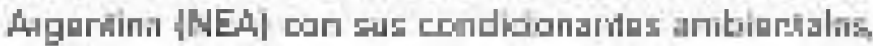

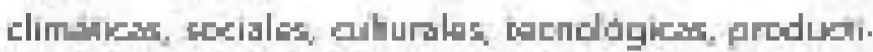

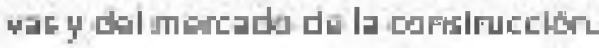

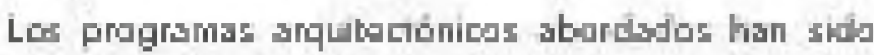
h.

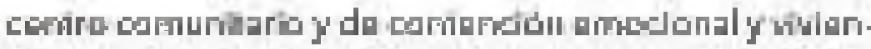
da da arrerganciz.

\section{Iniroducrín:}

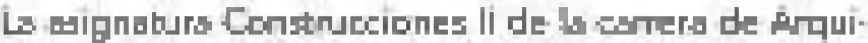

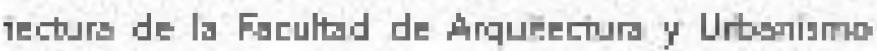

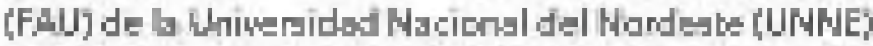

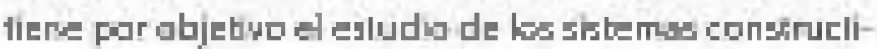
mos racianalaudas e indułialiados. Las matancis de

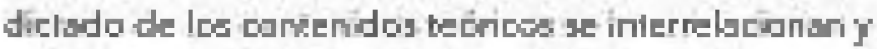
etruciuran slradedor de la actuidad próctica, que sonsiole en el daéro un sislema oongructibs no colunenganal.

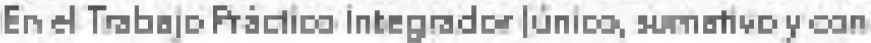

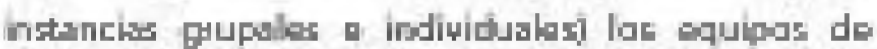

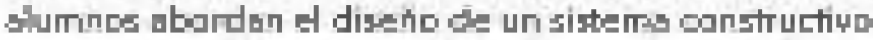

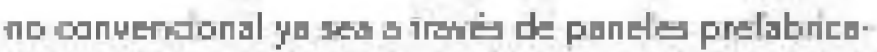

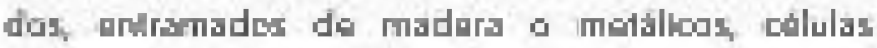

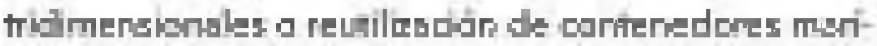
limpe

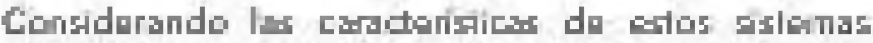
bonsiruclings que se manlan ers seca, en caras plaxus

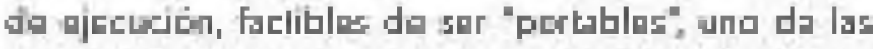

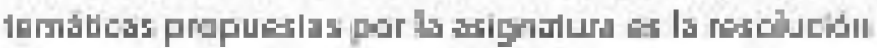
da copupamiantos para suidretrof́ns gancradas por

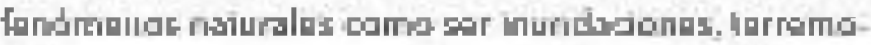

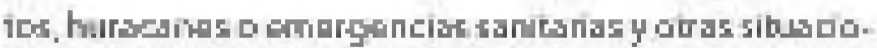

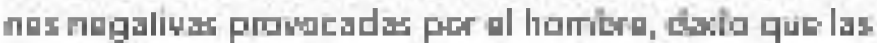
mignas requiaram da respuesias inmodiatos impori-

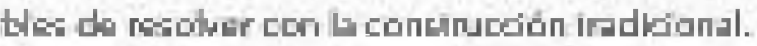

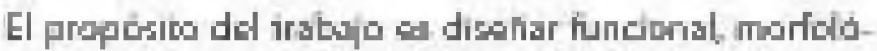
gica y en aspocial, ícenakgicaine-nte, diwarsas alternailus que a

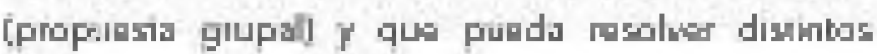


programas arquitectónicos (propuesta individual) como ser: hospital móvil, comedor comunitario, centro educativo, centro comunitario y de contención emocional y vivienda de emergencia.

En el ciclo lectivo 2015 la temática del riesgo ambiental (sus medidas de prevención, estudio y mitigación), ha sido abordada tanto desde el punto de vista teórico (como unidad temática de la asignatura), como desde el punto de vista práctico, dado que se constituye en el "problema de diseño" del TPl, e intenta incorporar los distintos aspectos de la realidad, con rasgos de práctica profesional anticipatoria, en la que una de las variables tenidas en cuenta es la incertidumbre intrinseca del proyecto arquitectónico. Además, se trata de sensibilizar al estudiante ante situaciones de vulnerabilidad tangibles dando respuestas potenciales y/o reales. El contexto de desarrollo es la región Nordeste de la Argentina (NEA) con sus condicionantes ambientales, climáticas, sociales, culturales, tecnológicas, productivas y del mercado de la construcción.

La propuesta didáctica tiene como antecedente la implementación del tema en la asignatura en el año 2010, atesorando logros y dificultades de aquella oportunidad, con una revisión, ajuste y adecuación permanente de los contenidos, las metodologias y estrategias de enseñanza - aprendizaje y las herramientas de evaluación.

\section{Objetivos:}

Los objetivos de la innovación pedagógica es la incorporación de temáticas motivadoras para que los alumnos comprendan el campo de aplicación de los sistemas constructivos no convencionales, sensibilizándolos con su futuro rol de profesionales comprometidos con el medio social y ambiental en el cual se desempeñarán.

Adicionalmente se incentiva el desarrollo de competencias para trabajar en equipo y comunicar resultados de su proceso de diseño al público objetivo, favore ciendo el proceso de síntesis.

El abordaje de esta temática como tema problema del TPI favorece la articulación teoría - práctica, no solo en los aspectos conceptuales de la asignatura, sino también actitudinales, favoreciendo la reflexión del alumno sobre del rol profesional en la construcción del hábitat y la intervención en ambientes vulnerables.

\section{Situaciones de catástrofe:}

Siguiendo a Natenzon, C. (1995) entendemos a los fenómenos naturales (terremotos, huracanes, inundaciones, sequías, deslizamientos de laderas, erupciones volcánicas, incendios, plagas) sujetos a distintas variables como ser su magnitud, duración, extensión, dispersión espacial, frecuencia o recurrencia, Lo que define un hecho como "desastroso" o "catastrófico" no es únicamente sus aspectos físico - naturales e incluye necesariamente el impacto en un grupo - sujeto - social vulnerable.

Además de los eventos calificados como "naturales" existen muchos otros originados por los propios seres humanos como incendios provocados, guerra, polución y contaminación química (OEA, 1993). En esta categoria podria incluirse también las situaciones de emergencia por enfermedades infecciosas tal es el caso de la pandemia de gripe H1N1 vivida como una emergencia de carácter global entre los años 2009 y 2010.

"El término amenaza es usado para describir el peligro latente que representa una amplia variedad de fenómenos; desde aquellos cuya ocurrencia se considera exclusivamente de origen natural, como terremotos, huracanes, erupciones volcánicas, etc., hasta aquellos cuyo origen se considera exclusivamente humano, tales como las guerras y los accidentes industriales. Entre estos dos extremos hay un amplio espectro de fenómenos, como, por ejemplo, hambrunas, inundaciones y deslizamientos, los cuales son provocados por la combinación de factores naturales y humanos" (Cardona, 1996, p. 59).

La vulnerabilidad social aparece como un aspecto central dado que un "peligro natural" se transforma en un "desastre natural" en la medida que causa un número inaceptable de muertes o daños a la propiedad (OEA, 1993), siendo determinante la exposición al riesgo. El crecimiento urbano irrestricto, en ambientes adversos o vulnerables, la falta de infraestructura y la falta de conciencia ambiental en las acciones cotidianas de amplias franjas poblacionales, agravan este escenario generando la exposición al riego de un alto número de habitantes.

Además de la propia situación de emergencia, con sus consecuencias inmediatas y concretas, es importante considerar las posibles consecuencias psicológicas negativas que desencadenan en la población y sus 
comunidades y que persiste aún luego de resuelta la situación, cuya cuantificación es prácticamente imposible.

\section{Implementación Didáctica:}

Estas situaciones adversas descriptas generan la necesidad de contar con equipamiento arquitectónico que permita sobrellevar la emergencia de la población afectada. Resulta característica la inmediatez de la necesidad, surgida de forma imprevista e improrrogable, por lo cual los plazos de la construcción tradicional resultan ineficaces.

Es justamente la construcción no convencional la que ofrece montajes en cortos plazos y la posibilidad de ser desmontada y montada en nuevos sitio, permitiendo la reutilización de los equipamientos.

El TPI se realiza en grupos de entre tres y cinco alumnos, alternando instancias grupales a individuales de diseño, en base a los siguientes sistemas constructivos:

- construcción prefabricada no integral de pequeñas, medianas o grandes piezas;

- construcción prefabricada integral (células tridimensionales y reutilización de contenedores);

- construcción en seco (ballom frame, steel frame).

El desafio es diseñar un sistema único de carácter grupal que luego pueda ser aplicado de forma individual a distintos programas arquitectónicos especificos, es decir, lograr la diversidad de expresiones individuales en la unicidad de criterios grupales.

Los programas arquitectónicos abordados han sido hospital móvil, comedor comunitario, centro educativo, centro comunitario y de contención emocional y vivienda de emergencia.

Además de tener en cuenta los aspectos funcionales, morfológicos y tecnológicos, se promueve desde el cuerpo docente la incorporación de criterios de sustentabilidad ambiental, como ser la utilización de energías renovables, el uso adecuado del agua, el diseño de la envolvente de acuerdo a un correcto estudio higrotérmico, utilización de materiales reutilizados o reciclados y la portabilidad del sistema que permitiría su uso en más de una situación, lo que significa una prolongación del ciclo de vida del mismo.

Las producciones realizadas por los estudiantes en la construcción no convencional, son el fruto de los conocimientos adquiridos con anterioridad en el ámbito de la tecnologia de los materiales y las técnicas constructivas, las cuales actúan como soporte necesario para plantear respuestas innovadoras con el desarrollo de diferentes sistemas constructivos, que estimulan la imaginación y la creatividad en el proyecto de soluciones concretas.

\section{Primera experiencias (ciclo lectivo 2010):}

Como consecuencia de la pandemia de gripe H1N1 y los Terremotos de Haiti y Chile ocurridos todos entre los años 2009 y principios de 2010 se propuso a los alumnos la indagación en la temática del equipamiento para situaciones de catástrofes.

La iniciativa fue muy bien recibida por los grupos de alumnos motivados por la utilidad de sus propuestas en este tipo de situaciones adveras. En la figura 1 se observa un panel sintesis de la comisión que desarrolla el trabajo en células tridimensionales, en este caso de hormigón.

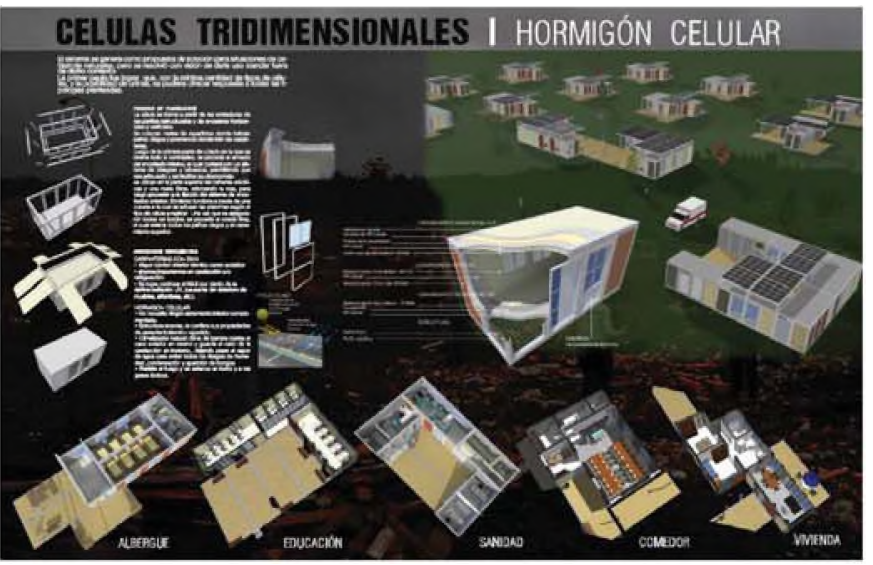

Figura 1: Panel Simteris del Grupo No 12 del ciclo lectwo 2010. Integrantes: Bianco, Maria Inǵs; Gover, Olivia; Pedroso, Maria Pia: Pizzorno, Javier y Sanz, Facundo. Docente a cargo: Mgter. Arq. Claudia Pilar.

\section{La propuesta implementada en el ciclo 2015 :}

Habiendo capitalizado la experiencia del año 2010, en base a su evaluación cualitativa y cuantitativa por parte del cuerpo docente, se propuso para el ciclo 2015 reiterar la temática, con resultados muy positivos, plasmados de forma elocuente en los TPI de los grupos de alumnos.

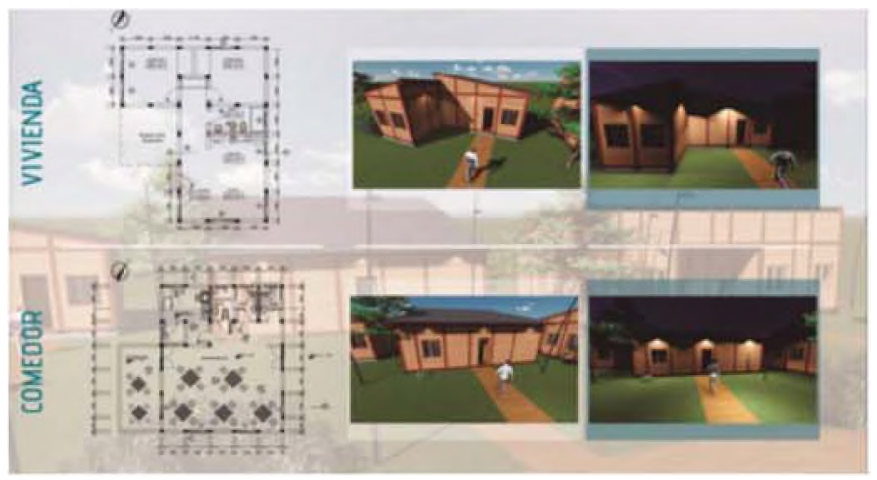




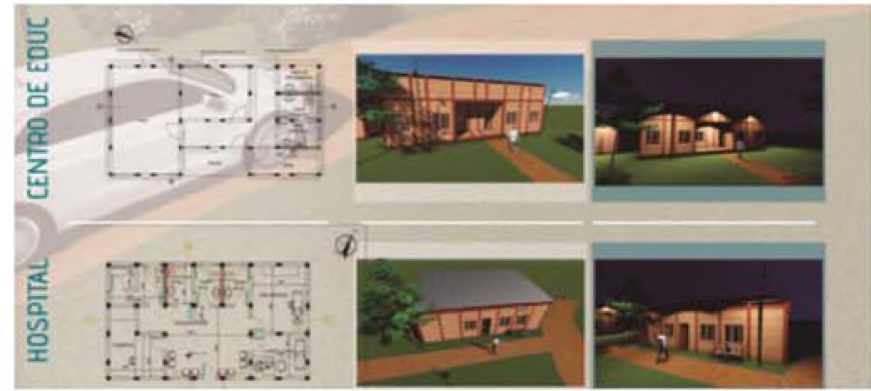

Figura 2: Una parte del panel Sintesis del Grupo $N^{0} 7$ del ciclo lectivo 2015 Integrantes: Botello, Estela Maria Inēs, Fachinı, Mgnacio, Valdez, Juliản Marcalo Wetzel, Federice. Docente a cargo: Mrg. Rasanna Gíselda Moran.

En la figura 2 puede observarse un sector del panel síntesis correspondiente a la comisión de trabajo de prefabricación no integral (paneles prefabricados) usando como material principal la madera, mientras que en la figura 3 se observa planillas sintesis de un equipo que desarrolló células tridimensionales en madera. Con dichas planillas se elabora un dossier anual para ser utilizado como material de consulta de los ciclos posteriores.
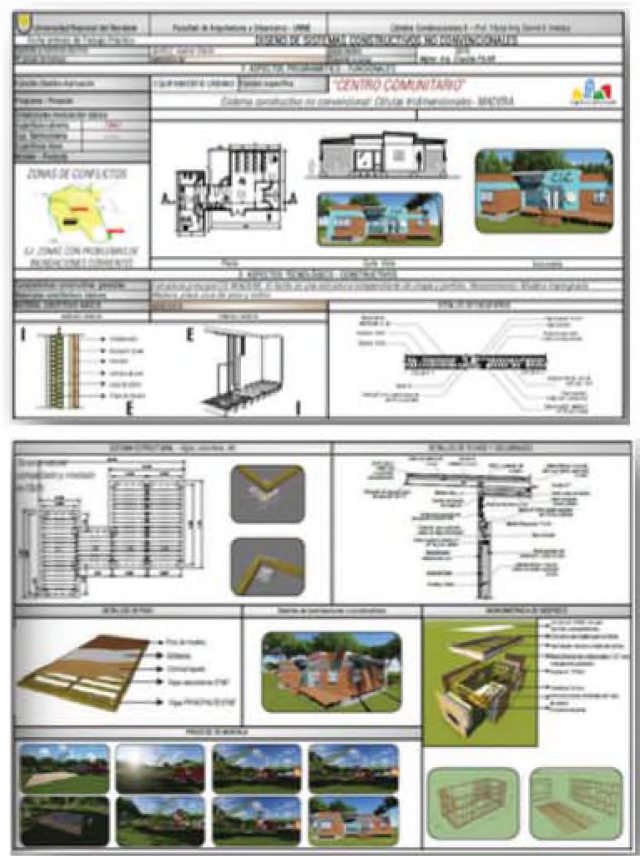

Figura 3: planillas sintesis del grupa 56 del ciclo lectivo 2015. Integrantes: Bóveda, Aristıdes; Cano, Leandro; Godoy, Fermando; Maciel, Marı y Ouiroz, Gabriel. Docente a cargo: Mgter. Arq. Claudia Pilaf.

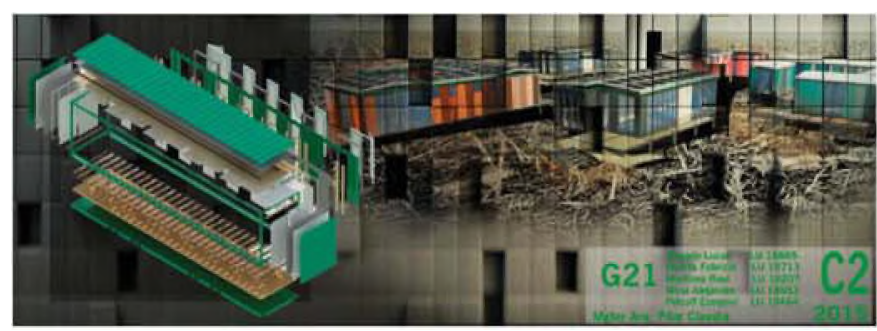

Figura 4: panel sintesis del grupo 21 del ciclo lactwa 2015. Integrantes: Bogado, Lueas; Duarte, Fabricio: Martinez, Oscas; Mına, Alejandro Nicolás y Petcoff, Ezequiel. Docente a cargo: Mgter. Arq. Claudia Pilar.
En la figura 4 puede observarse un sector del panel síntesis de un equipo que realizó el TPI reutilizando contenedores. A la izquierda puede verse un despiece tecnológico de las distintas capas y materiales utilizados y a la derecha un fotomontaje que refleja el espíritu del tema abordado en el ciclo lectivo 2015 sobre equipamientos para afrontar situaciones de catástrofes ambientales.

\section{El enfoque pedagógico:}

El desarrollo de la asignatura se articula en un Trabajo Práctico Integrador(TPI) que favorece especialmente el aprendizaje de los contenidos procedimentales. Su planteo, al inicio del cursado, sitúa al alumno en una condición de carencia, en la cual reconoce sus propios limites y hace que el desarrollo de los temas teóricos sea internalizados y acomodados de una forma más eficaz. En algunos puntos del desarrollo de la asignatura "la práctica antecede a la teoría", con el objetivo de favorecer el aprendizaje significativo sobre todos de los contenidos procedimentales.

La motivación del trabajo se da atendiendo los intereses del estudiante en la elaboración de propuestas destinadas a resolver problemas reales con la posibilidad de investigar sobre temas del entorno social en el que se desenvuelve y desarrollar habilidades requeridas para un eficiente desempeño profesional en el siglo XXI.

Lo interesante de la mencionada propuesta es que resulta motivadora dentro del proceso de enseñanza aprendizaje sosteniéndose durante todo el ciclo lectivo cursado, manteniendo al alumno activo y predispuesto a la incorporación de nuevos conocimientos.

Desde un punto de vista teórico el trabajo de diseño se enmarca en el "Método de Desarrollo de Proyectos". En todo momento se intenta que la realidad sea el marco del TPI, iniciándolo en un "análisis de mercado" referido a los materiales y sistemas constructivos que se desarrollarán durante la asignatura. Durante la actividad los estudiantes buscan información en: la web, comercios, corralones de materiales de construcción; entrevistan a comerciantes y profesionales; recopilan datos que luego sintetizan, organizan y vierten en un informe, el que servirá de insumo para la elaboración del TPI. En esta etapa se considera como enriquecedor el hecho de la labor grupal y colaborativa, además del intercambio de la información con los demás grupos. Una de las caracteristicas del Método de Desarrollo de 
Proyectos es que permite establecer un vinculo distinto entre docentes y estudiantes, centrados en la resolución de un problema práctico con posibles impactos en la sociedad, al mismo tiempo que se favorecen acciones colaborativas entre los distintos grupos de trabajo. En este contexto el docente asume el rol de "animador" o "facilitador" del proceso de aprendizaje entendiendo que el estudiante está próximo a ser profesional y uno de los propósitos de la asignatura es colaborar en el proceso de integración de conocimientos adquiridos en el desarrollo de la carrera, tanto de tipo conceptuales como procedimentales y actitudinales.

El Método de Desarrollo de Proyectos es una herramienta enriquecedora que les permite a los estudiantes involucrarse en sus propios aprendizajes de forma distinta, convirtiéndolos en verdaderos protagonistas del proceso, activando el aprendizaje de habilidades y contenidos por medio de una enseñanza socializada. Cuando se utiliza el método de proyectos como estrategia, los estudiantes estimulan sus habilidades más fuertes y desarrollan algunas nuevas. Se motiva en ellos el interés por el aprendizaje, un sentimiento de responsabilidad y esfuerzo y un entendimiento del rol tan importante que tienen en sus comunidades.

\section{El proceso de evaluación:}

Construcciones II es la última asignatura de la serie tecnológico-constructiva con que cuenta la carrera de arquitectura, por lo cual se trata de implementar una actividad integradora en la que se ponen en juego todos los conocimientos alcanzados en las experiencias curriculares recorridas.

El trabajo es prácticamente de tipo autónomo basado en la idea de coaching o entrenamiento, permitiendo un rol más activo por parte del estudiante, encarando desafíos y resolviendo problemas dentro de un grupo donde deberá desprenderse de idea adquiridas previamente y respetar la de los otros, logrando un consenso como equipo.

En el transcurrir de la asignatura el alumno parte de una situación de "novato" (en la que la autonomía, la automatización y la eficacia y eficiencia de su desempeño es aún muy limitado) a una situación que se acerca a la del "experto", intentando incluir contextos de incertidumbre propios de la realidad.

Las actividades planteadas por la cátedra exigen una alta dedicación del estudiante, quien debe poner a prueba el conjunto de destrezas que fue adquiriendo en el transcurso de la carrera, para poder resolver la problemática propuesta. Rescatando las habilidades de diseño y la creatividad, fundamentales en el proceso. Compatibilizar resoluciones tecnológico- constructivas novedosas y creativas resulta realmente complejo a la hora de la toma de decisiones en el proceso proyectual.

Algunos grupos de trabajo, de forma voluntaria, realizan videos sobre los procesos de producción (fabricación) y montaje propuestos. Este medio de comunicación resulta especialmente eficaz para los alumnos de años posteriores, que se entusiasman con la posibilidad de integrar diseño tecnológico, creatividad y animación digital.

Además se puede inferir que las actividades propuestas resultan atractivas para los estudiantes, dado que un alto número de ellos proponen temáticas relacionadas al momento de realizar su Trabajo Final de Carrera (en sexto año) con el objeto de profundizar sus conocimientos y realizar una experiencia real, potencialmente transferible al medio social, político y económico de la región.

La asignatura ofrece la posibilidad de una promoción directa, sin examen final, mediante la aprobación con más de 8 puntos de las dos pruebas parciales (con posibilidad de un recuperatorio) más la aprobación del TPI (para la totalidad de los cursantes), aquellos alumnos que no alcancen dicha promoción, pasan al sistema de regularidad y debe aprobar un examen final de carácter sumativo.

Carrizo, (2009) expresa que "la necesidad de saber", de tener información acerca de los aprendizajes de los alumnos, es la que nos lleva a plantear diversos momentos y diversos instrumentos. Es así que se plantean evaluaciones: de diagnóstico, de seguimiento y de acreditación parcial o final.

Los objetivos pedagógicos aplicados en cada una de las evaluaciones permiten lograr un concepto general del alumno y del grupo de alumnos. De esta manera la evaluación diagnóstica realizada al inicio del curso, nos brinda un primer indicador de los conocimientos previos del grupo y de cada uno de los alumnos. Las evaluaciones formativas o de seguimiento nos permiten evaluar el avance del proceso y la sumativa, el resultado (de acreditación parcial o final) referida a los exámenes parciales o finales que pretenden cerrar etapas en el aprendizaje. Esta evaluación será mucho 
más útil si el alumno puede aprovecharla como otra instancia para aprender y no como un obstáculo a sortear para obtener aprobar. (Carrizo, 2009).

También somos conscientes que el aprendizaje de esta asignatura no culmina el día de la evaluación final del alumno, sino que dado la diversidad de experiencias profesionales o los mecanismos personales de aprendizaje muchos conocimientos serán reforzados a partir de su aplicación e integrado a sus herramientas cognitivas quizá de manera no previstas por la asignatura, dado que posee un gran bagaje de conceptos(que puede ser transferido a nuevas situaciones ya sean estas de carácter tecnológico, de diseño, funcional, de estético, etc.)

En la actualidad se valora el aprendizaje del alumno en el proceso y en el producto. La incidencia de estos dos aspectos en la enseñanza reglada queda claramente reflejado por las diversas normas que existen alrededor de este tema; por las incidencias en la planificación del trabajo del profesorado, en la actividad en el aula y en la actividad reflexiva posterior. La evaluación sumativa y formativa está presente en toda planificación escolar, en toda programación, en la misma aula. (Bordas y Cabrera, 2001)

Carrizo (2009) admite la gran responsabilidad que nos cabe a los docentes formadores al momento de plantear la evaluación. Reflexionando sobre lo siguiente: Los profesionales que reciban su título deberán ser capaces de desempeñarse en sus actividades con los conocimientos, habilidades y destrezas que nosotros hayamos enseñado.

\section{Conclusiones:}

Los sistemas constructivos no convencionales, por sus características tecnológicas, resultan altamente adecuados para dar respuestas rápidas en situaciones de catástrofe, ya sean éstas consecuencias de procesos físicos - naturales o provocadas o amplificadas por el hombre.

La experiencia didáctica en los ciclos lectivos 2010 y 2015 de la asignatura Construcciones II permitieron verificar esta hipótesis de trabajo, con resultados altamente satisfactorios de los diseños propuestos por los estudiantes.

Tomando en cuenta la vertiginosidad de los cambios tecnológicos dados en la actualidad, nos encontramos con propuestas altamente superadoras, tanto en el diseño arquitectónico como en la creatividad de las técnicas constructivas no convencionales.

De esta manera la problemática ambiental articula teoría y práctica, y permite a los estudiantes desarrollar un proyecto para mejorar su contexto y los involucra en la construcción de un ambiente más sustentable como futuros profesionales.

Además, de los nuevos conocimientos adquiridos en la asignatura, el uso de Métodos de Desarrollo de Proyectos incentiva a los estudiantes a continuar con la investigación y profundización de temas de carácter interdisciplinar vinculados al mundo real, trascendiendo el aula de clases.

Asimismo se promueve un proceso que fortalece los vinculos entre los estudiantes, ya sean de la misma promoción o de ciclos posteriores, dado que los trabajos de los alumnos se compilan para ser utilizados como material de consulta, paliando cierta carencia de bibliografía actualizada, de difícil o costoso acceso.

\section{Referencias:}

-ALÍAS, H., MORÁN, R., PILAR, C., SCHUSTER, A. y VEDOYA, D. Criterios de sustentabilidad en el diseño de viviendas mediante sistemas constructivos no convencionales: experiencias en la enseñanza. XXIX Encuentro y XIV Congreso ARQUISUR, Tarija, Bolivia. 2010

-ALÍAS, H., PILAR, C. y VEDOYA, D. Articulación teoría práctica en la enseñanza de la construcción no convencional. La experiencia de la cátedra "Construcciones II" de la Facultad de Arquitectura y Urbanismo de la UNNE. Cuartas Jornadas Comunicación de Experiencias Pedagógicas Innovadoras. Programa de Formación Docente Continua. Secretaria General Académicas. UNNE, 17 y 18 de noviembre de 2011. Actas publicadas en formato digital. ISBN 978-950-656-139-0. 2011 -BORDAS, M. Inmaculada; CABRERA, Flor, Estrategias de evaluación de los aprendizajes centrados en el proceso. Departamento de didáctica y organización educativa. Departamento de Métodos de investigación y diagnóstico en educación. Universidad de Barcelona, Revista Española de Pedagogía. Año LIX, enero-abril, $N^{\circ} 218$. pp. 25 a 48.2001

-CARDONA, Omar D. Manejo Ambiental y prevención de desastres: dos temas asociados. En Ciudades en riesgo. Degradación ambiental, riesgos urbanos y desastres. Red de Estudios Sociales en Prevención de Desastres en América Latina. LA RED. USAID. Fernández, María Augusta (Comp.). 1996 


\section{AT | Revista ARQUITECNO | N8}

-CARRIZO, Walter. La responsabilidad del docente frente a la evaluación. Univ. Nac. de la Patagonia San Juan Bosco. Fac. Ciencias Económicas. Comodoro Rivadavia - Chubut (Argentina) 2009

-COSTA DURAN, Sergi. New Prefab. Reeditar Libros. 2009

-Dirección de Investigación y Desarrollo Educativo. Vicerrectoría Académica, Instituto Tecnológico y de Estudios Superiores de Monterrey, México. El método de proyectos como técnica didáctica. En "Las estrategias y técnicas didácticas en el rediseño". Disponible en http://www.sistema.itesm.mx/va/dide/inf-doc/estrategias/.

-MAC DONNELL, H. y MAC DONNELL, H. P. Manual de Construcción Industrializada. I.S.B.N. 987-97522-3-6. REVISTA VIVIENDA SRL. Buenos Aires. Argentina. 2004 -MARTÍ, J. A.; HEYDRICH, M.; ROJAS, M.; HERNÁNDEZ, A. Aprendizaje basado en proyectos: una experiencia de innovación docente. Revista Universidad EAFIT, vol. 46, núm. 158, abril-junio, 2010, pp. 11-21. Universidad EAFIT. Medellin, Colombia. 2010.

-MIGUET, Josep M (Ed.) Prefab Design. Ed. Monsa. 2006.

-NATENZON, Claudia. Catástrofes naturales, riesgo e incertidumbre. FLACSO. Serie Documentos e Informes de Investigación N 197. Buenos Aires, Argentina. 1995. -OEA. Manual sobre el manejo de peligros naturales en la planificación para el desarrollo regional integrado. Washington, D.C. Dpto. de Desarrollo Regional y Medio Ambiente, Secretaria Ejecutiva para Asuntos Económicos y Sociales. 1993

-PILAR, C., VEDOYA, D. y KOZAK, N. Construcción NO Convencional: Las Células Tridimensionales como alternativa para el diseño de equipamientos con criterios de sustentabilidad ambiental. 60 Congreso Regional de Tecnología de las Facultades del -ARQUISUR. Instituto de Tecnología Arquitectónica. Facultad de Arquitectura y Urbanismo. Universidad Nacional de Tucumán. Tucumán, Argentina. 2013.

-PILAR,C, VEDOYA, D., KOZAK, N. Resolución de equipamientos para el espacio público mediante sistemas constructivos no convencionales. Revisa Arquitecno. Ediciones del ITDAHu, Corrientes. 2015

VEDOYA, D. La tecnología Nuestra de Cada Día. Corrientes: Ediciones del I.T.D.A.Hu. (F.A.U.-U.N.N.E.) 2001. 